Proyecciones Journal of Mathematics

Vol. 38, No 2, pp. 203-219, June 2019.

Universidad Católica del Norte

Antofagasta - Chile

\title{
The integral sine addition law
}

\author{
D. Zeglami \\ Moulay Ismail University, Morocco \\ M. Tial \\ Ibn Tofail University, Morocco \\ and \\ S. Kabbaj \\ Ibn Tofail University, Morocco \\ Received : February 2017. Accepted : January 2019
}

\begin{abstract}
In the present paper we determine, in terms of characters and additive functions, the solutions of the integral functional equation for the sine addition law

$$
\int_{G} f(x y t) d \mu(t)=f(x) g(y)+g(x) f(y), x, y \in G,
$$

where $G$ is a locally compact Hausdorff group and $\mu$ is a regular, compactly supported, complex-valued Borel measure on G. Some consequences of this result and an example are presented.
\end{abstract}

Subjclass: 39B32, 39B52.

Keywords: Functional equation, Sine and cosine addition laws, Character, Borel measure. 


\section{Introduction}

Let $G$ be a topological group with neutral element $e$. The trigonometric addition and subtraction formulas have been studied in the context of functional equations by a number of mathematicians. The monographs by Aczél [1], by Kannappan [6], by Stetkær [8] and by Székelyhidi [11] have references and detailed discussions of the classic results.

Chung, Kannappan and $\mathrm{Ng}$ [2] solved on any group $G$, the functional equation

$$
f(x y)=f(x) g(y)+f(y) g(x)+h(x) h(y), \quad x, y \in G .
$$

Poulsen and Stetkær [7] found the complete set of continuous solutions of each of the functional equations

$$
\begin{aligned}
& g(x y)=g(x) g(y)-f(x) f(y), x, y \in G, \\
& f(x y)=f(x) g(y)+f(y) g(x), x, y \in G .
\end{aligned}
$$

In the paper [12], Van Vleck studied the continuous solutions $f: \mathbf{R} \rightarrow \mathbf{R}$ of the functional equation

$$
f\left(x-y+z_{0}\right)-f\left(x+y+z_{0}\right)=2 f(x) f(y), \quad x, y \in \mathbf{R},
$$

where $z_{0}>0$ is fixed. Extensions of (1.3) and related functional equations from $\mathbf{R}$ to locally compact groups can be found e.g. in $([4,5,9,13,14,15])$. Results of [10] where Stetkær find the solutions of the cosine addition law with an additional term

$$
g(x y)=g(x) g(y)-f(x) f(y)+\alpha f(x y),
$$

(on semigroups) for a fixed complex constant $\alpha$, and of [12] have been an inspiration for this work in their treatments of (1.4) and (1.3), respectively.

To formulate our results we introduce the following notations and assumptions that will be used throughout the paper: $C(G)$ denotes the algebra of continuous, complex valued functions on $G$. The set of homomorphisms $a: G \rightarrow(\mathbf{C},+)$ will be called the additive maps and denoted by $\mathcal{A}(G)$.

A character $\chi$ of $G$ is a homomorphism $\chi: G \rightarrow \mathbf{C}^{*}$, where $\mathbf{C}^{*}$ denotes the multiplicative group of non-zero complex numbers. So characters need not be unitary in the present paper. It is well known that the set of 
characters on $G$ is a linearly independent subset of the vector space of all complex-valued functions on $G$ (see [8, Corollary 3.20]).

Let $G$ be a locally compact Hausdorff group, and let $M_{C}(G)$ denote the space of all regular, compactly supported, complex-valued Borel measures on $G$. For $\mu \in M_{C}(G)$, we use the notation

$$
\mu(f)=\int_{G} f(t) d \mu(t),
$$

for all $f \in C(G)$.

Let $\mu \in M_{C}(G)$. The purpose of the present paper is to give an explicit description of the continuous solutions $f, g: G \rightarrow \mathbf{C}$ of the following integral version of the sine addition law

$$
\int_{G} f(x y t) d \mu(t)=f(x) g(y)+g(x) f(y), x, y \in G .
$$

To solve Eq. (1.5) we reduce it to Eqs. (1.1), (1.2) and the following functional equation

$$
f(x y)=f(x) g(y)+g(x) f(y)-g(x y), \quad x, y \in G,
$$

the continuous solutions of which, in terms of characters and additive functions, are given in Theorem 2.1.

As an important consequence, we solve the following functional equation

$$
f\left(x y z_{0}\right)=f(x) g(y)+g(x) f(y), \quad x, y \in G,
$$

where $z_{0} \in G$ is an arbitrarily fixed element. Eq. (1.6) results from (1.5) by replacing $\mu$ by $\delta_{z_{0}}$ : the Dirac measure concentrated at $z_{0}$.

Let $n \in \mathbf{N}, \alpha_{1}, \cdots, \alpha_{n} \in \mathbf{C}$, and $z_{1}, \cdots, z_{n} \in G$ be arbitrarily fixed elements. As a more general consequence of our result, we obtain all continuous solutions of the following version of the sine addition formula

$$
\sum_{i=1}^{n} \alpha_{i} f\left(x y z_{i}\right)=f(x) g(y)+g(x) f(y), \quad x, y \in G .
$$

In Proposition 2.6 we characterize the solutions of the integral functional equation for the sine subtraction law

$$
\int_{G} f(x y t) d \mu(t)=f(x) g(y)-g(x) f(y), x, y \in G .
$$

In the last section, we provide a concrete example to show that nontrivial continuous solutions of (1.6) occur in real life. 
Note that the following integral versions of the addition and subtraction formulas for cosine and sine:

$$
\begin{aligned}
\int_{G} g(x y t) d \mu(t) & =g(x) g(y)-f(x) f(y), x, y \in G, \\
\int_{G} f(x \sigma(y) t) d \mu(t) & =f(x) g(y)-g(x) f(y), x, y \in G,
\end{aligned}
$$

where $\sigma$ denotes an involution of $G$, i.e., $\sigma(x y)=\sigma(x) \sigma(y)$ and $\sigma(\sigma(x))=x$ for all $x, y \in G$, were solved in [16].

\section{The solutions of the integral sine addition law}

The purpose of this section is first to give an explicit description of the solutions of the functional equation

$$
f(x y)=f(x) g(y)+g(x) f(y)-g(x y), x, y \in G,
$$

where $f, g: G \rightarrow \mathbf{C}$ are unknown functions. And secondly to determine the solutions $f, g: G \rightarrow \mathbf{C}$ of the functional equation (1.5), namely

$$
\int_{G} f(x y t) d \mu(t)=f(x) g(y)+g(x) f(y), x, y \in G,
$$

where $\mu \in M_{C}(G)$, in terms of characters and additive maps of $G$.

In the following theorem we exhibit the solutions of the functional equation (2.1) which were solved by Chung, Kannappan and $\mathrm{Ng}[2]$, but their results were formulated differently from what we need. So we present, using [10, Theorem 6.3], a modified proof of the solution formulas.

Theorem 2.1. Let $G$ be a topological group. The pair $f, g \in C(G)$ is a solution of the functional equation (2.1) if and only if one of the possibilities listed below takes place:

(i) There exist a constant $q \in \mathbf{C}$ and a continuous character $\chi$ of $G$ such that

$$
f=(1-q+\delta) \frac{\chi}{2} \quad \text { and } \quad g=(1+q+\delta) \frac{\chi}{2},
$$

where $\delta= \pm \sqrt{1+q^{2}}$. 
(ii) There exist a constant $q \in \mathbf{C}^{*}$ and two different continuous characters $\chi_{1}$ and $\chi_{2}$ of $G$ such that

$f=\frac{\chi_{1}+\chi_{2}}{2}+(\delta-q) \frac{\chi_{1}-\chi_{2}}{2}$ and $g=\frac{\chi_{1}+\chi_{2}}{2}+(\delta+q) \frac{\chi_{1}-\chi_{2}}{2}$,

where $\delta= \pm \sqrt{1+q^{2}}$.

(iii) There exist a continuous character $\chi$ of $G$ and a non-zero continuous and additive function $a: G \rightarrow \mathbf{C}$ such that

$$
f=\chi \text { and } g=\chi(1+a) \text { or } f=(1+a) \chi \text { and } g=\chi \text {. }
$$

Proof. We define the function $H:=\frac{1}{2}(f+g)$. Then the equation (2.1) becomes

$$
H(x y)=-(g(x)-H(x))(g(y)-H(y))+H(x) H(y), x, y \in G .
$$

Then we obtain the functional equation

$$
H(x y)=H(x) H(y)-K(x) K(y), x, y \in G,
$$

where $K:=g-H$, the solutions of which were given in [10, Theorem 6.3]. Applying [10, Theorem 6.3] we infer that there are only the following cases:

(i) There exist a constant $q \in \mathbf{C}$ and a continuous character $\chi$ of $G$ such that

$$
K=q \frac{\chi}{2} \text { and } H=\left(1 \pm \sqrt{1+q^{2}}\right) \frac{\chi}{2},
$$

which shows that

$$
f=(1-q+\delta) \frac{\chi}{2} \quad \text { and } \quad g=(1+q+\delta) \frac{\chi}{2},
$$

where $\delta= \pm \sqrt{1+q^{2}}$.

(ii) There exist a constant $q \in \mathbf{C}^{*}$ and two different continuous characters $\chi_{1}$ and $\chi_{2}$ of $G$ such that

$$
K=q \frac{\chi_{1}-\chi_{2}}{2} \text { and } H=\frac{\chi_{1}+\chi_{2}}{2} \pm \sqrt{1+q^{2}} \frac{\chi_{1}-\chi_{2}}{2},
$$

which gives 


$$
f=\frac{\chi_{1}+\chi_{2}}{2}+(-q+\delta) \frac{\chi_{1}-\chi_{2}}{2} \text { and } g=\frac{\chi_{1}+\chi_{2}}{2}+(q+\delta) \frac{\chi_{1}-\chi_{2}}{2},
$$

where $\delta= \pm \sqrt{1+q^{2}}$.

(iii) There exist a continuous character $\chi$ of $G$ and a non-zero continuous function $A \in \mathcal{A}(G)$ such that

$$
K=\chi A \quad \text { and } H=\chi(1 \pm A)
$$

and so we obtain

$$
f=\chi(1-A+\phi) \quad \text { and } \quad g=\chi(1+A+\phi),
$$

where $\phi= \pm A$. Then $f=\chi$ and $g=\chi(1+a)$ or $f=(1-a) \chi$ and $g=\chi$ where $a:=2 A$.

Conversely, simple computations prove that the formulas above for $f$ and $g$ define solutions of $(2.1)$.

Remark 2.2. i) The continuity of characters and the additive functions in [10, Theorem 6.3] and consequently in Theorem 2.1 results from [10, Proposition 5.2].

ii) Eq. (2.1) is symmetric i.e. if the pair of functions $(\varphi, \psi)$ is a solution of $(2.1)$ then $(\psi, \varphi)$ is also a solution of (2.1). This is clearly seen in the form of solutions in Theorem 2.1.

Now we are in the position to describe all solutions of the functional equation (2.2).

Theorem 2.3. Let $G$ be a locally compact Hausdorff group and $\mu \in$ $M_{C}(G)$. Assume that the pair $f, g \in C(G)$ is a solution of Eq. (2.2). Then we have the following possibilities:

1. $g=0$ and $f$ is any function in $C(G)$ such that $\int_{G} f(\cdot t) d \mu(t)=0$ or $f=0$ and $g$ is arbitrary in $C(G)$.

2. There exist a constant $\gamma \in \mathbf{C}^{\star}$ and a continuous character $\chi$ of $G$, with $\mu(\chi) \neq 0$, such that

$$
f=\gamma \chi \text { and } g=\frac{\mu(\chi)}{2} \chi .
$$


3. There exist a constant $\gamma \in \mathbf{C}^{\star}$ and two continuous characters $\chi_{1}$ and $\chi_{2}$ of $G$, with $\mu\left(\chi_{2}\right)=-\mu\left(\chi_{1}\right) \neq 0$, such that

$$
f=\frac{\gamma}{2}\left(\chi_{1}+\chi_{2}\right) \text { and } g=\frac{\mu\left(\chi_{1}\right)}{2}\left(\chi_{1}-\chi_{2}\right) .
$$

4. There exist a constant $c \in \mathbf{C}^{\star}$ and two different continuous characters $\chi_{1}$ and $\chi_{2}$ of $G$, with $\mu\left(\chi_{2}\right)=\mu\left(\chi_{1}\right) \neq 0$, such that

$$
f=c\left(\chi_{1}-\chi_{2}\right) \text { and } g=\frac{\mu\left(\chi_{1}\right)}{2}\left(\chi_{1}+\chi_{2}\right) .
$$

5. There exist constants $\gamma, \beta, q \in \mathbf{C}^{\star}$ and two continuous characters $\chi_{1}$ and $\chi_{2}$ of $G$, with $\mu\left(\chi_{1}\right)=\beta \frac{2+2 \delta}{1-q+\delta}$ and $\mu\left(\chi_{2}\right)=\beta \frac{2-2 \delta}{1+q-\delta}$, such that

$$
\begin{aligned}
f & =\gamma\left\{\frac{\chi_{1}+\chi_{2}}{2}+(-q+\delta) \frac{\chi_{1}-\chi_{2}}{2}\right\} \\
\text { and } g & =\beta\left\{\frac{\chi_{1}+\chi_{2}}{2}+(q+\delta) \frac{\chi_{1}-\chi_{2}}{2}\right\},
\end{aligned}
$$

where $\delta:= \pm \sqrt{1+q^{2}}$.

6. There exist a non-zero continuous function $a \in \mathcal{A}(G)$ and a continuous character $\chi$ of $G$, with $\mu(a \chi)=0$ and $\mu(\chi) \neq 0$, such that

$$
f=\chi a \text { and } g=\mu(\chi) \chi
$$

7. There exist a constant $\gamma \in \mathbf{C}^{\star}$, a continuous function $a \in \mathcal{A}(G)$ and a continuous character $\chi$ of $G$, with $\mu(\chi)=\mu(a \chi) \neq 0$, such that

$$
f=\gamma(1+a) \chi \quad \text { and } g=\mu(\chi) \chi
$$

Conversely, the formulas above for $f$ and $g$ define solutions of (2.2).

Proof. Let the pair $f, g$ be a solution of the equation (2.2). Letting $y=e$ in $(2.2)$ we get that

$$
\int_{G} f(x t) d \mu(t)=\beta f(x)+\gamma g(x), x \in G,
$$


where $\beta=g(e)$ and $\gamma=f(e)$. So, using (2.3), we can reformulate the form of Eq. (2.2) as

$$
\beta f(x y)=f(x) g(y)+g(x) f(y)-\gamma g(x y), x, y \in G .
$$

Case 1: Suppose that $\beta=0$ then (2.4) gives

$$
\gamma g(x y)=f(x) g(y)+g(x) f(y) \quad x, y \in G .
$$

If $\gamma=0$ then equation (2.4) becomes

$$
f(x) g(y)=-g(x) f(y) x, y \in G,
$$

thus by a small computation we show that we are in the case (1) of our statement.

If $\gamma \neq 0$ then from (2.4) we obtain the functional equation

$$
g(x y)=F(x) g(y)+g(x) F(y), x, y \in G,
$$

where $F=\frac{1}{\gamma} f$, the solutions of which were given in [7, Proposition III.1]. We work our way through the possibilities (a)-(d) presented by [7, Proposition III.1] to see what the properties (2.3), that $g(e)=0$ and $f(e) \neq 0$ entail. We use the notation of [7, Proposition III.1] with $m$ replaced here by $\chi$.

(a) $g=0$ and $f$ is any continuous function. Using (2.3) we get that $\int_{G} f(\cdot t) d \mu(t)=0$. So we are in $(1)$.

(b) $g=c \chi$ and $f=\gamma \frac{\chi}{2}$. Here we have $f(e)=\gamma=\frac{\gamma}{2}$, which implies that $\gamma=0$. So this case is excluded.

(c) $g=c\left(\chi_{1}-\chi_{2}\right)$ and $f=\frac{\gamma}{2}\left(\chi_{1}+\chi_{2}\right)$. If $c=0$ or $\chi_{1}=\chi_{2}$ then $g=0$ so we are in (1). Otherwise, using (2.3) and the linear independence of different characters we infer that $\mu\left(\chi_{1}\right)=2 c$ and $\mu\left(\chi_{2}\right)=-2 c$. So we are in $(3)$.

(d) $g=\chi a$ and $f=\gamma \chi$. Using (2.3) we get $a=\mu(\chi)=0$ then $g=0$. So we are in (1).

Case 2: Suppose that $\beta \neq 0$. We can reformulate the form of Eq. (2.4) as

$$
f(x y)=f(x) G(y)+f(y) G(x)+\gamma G(x y), x, y \in G,
$$

where $G:=\frac{1}{\beta} g$, the solutions of which, in the subcase $\gamma=0$, are given in [7, Proposition III.1]. When we analyse them we find, using the notation in [7, Proposition III.1] with $m$ replaced here by $\chi$, that: 
(a) $f=0$ and $g$ is any function. So we are in (1).

(b) $f=c \chi$ and $g=\beta \frac{\chi}{2}$. Here we have $g(e)=\beta=\frac{\beta}{2}$, which implies that $\beta=0$. So this case is excluded.

(c) $f=c\left(\chi_{1}-\chi_{2}\right)$ and $g=\frac{\beta}{2}\left(\chi_{1}+\chi_{2}\right)$. If $c=0$ or $\chi_{1}=\chi_{2}$ then $f=0$ so we are in (1) and otherwise a computation based on (2.3) and the linear independence of different characters shows that

$$
c \mu\left(\chi_{1}\right)=c \beta \text { and } c \mu\left(\chi_{2}\right)=c \beta,
$$

which gives $\mu\left(\chi_{1}\right)=\mu\left(\chi_{2}\right)=\beta \neq 0$ thus we are in (4).

(d) $f=\chi a$ and $g=\beta \chi$. Using (2.3) we get that $\mu(\chi)=\beta \neq 0$ and $\mu(a \chi)=0$. This requires $a \neq 0$. So we are in (6).

For the subcase $\gamma \neq 0$, the equation (2.4) becomes

$$
F(x y)=F(x) G(y)+G(x) F(y)-G(x y) x, y \in G,
$$

where $G=\frac{1}{\beta} g$ and $F=\frac{1}{\gamma} f$. Applying Theorem 2.1 we infer that there are only the following 3 cases:

(i) There exist a constant $q \in \mathbf{C}$ and a continuous character $\chi$ of $G$ such that

$$
f=\gamma(1-q+\delta) \frac{\chi}{2} \quad \text { and } \quad g=\beta(1+q+\delta) \frac{\chi}{2},
$$

where $\delta= \pm \sqrt{1+q^{2}}$. Since $f(e)=\gamma$ and $g(e)=\beta$ then $1-q+\delta=$ $1+q+\delta=2$ (i.e $q=0$ and $\delta=1$ ) and so $f=\gamma \chi$ and $g=\beta \chi$. Using (2.3) we get that $\mu(\chi)=2 \beta \neq 0$. So we are in the case (2) of our statement.

(ii) There exist a constant $q \in \mathbf{C}^{*}$ and two different continuous characters $\chi_{1}$ and $\chi_{2}$ of $G$ such that

$$
\begin{aligned}
f & =\gamma\left(\frac{\chi_{1}+\chi_{2}}{2}+(-q+\delta) \frac{\chi_{1}-\chi_{2}}{2}\right) \\
& =\gamma(1-q+\delta) \frac{\chi_{1}}{2}+\gamma(1+q-\delta) \frac{\chi_{2}}{2}
\end{aligned}
$$

and

$$
\begin{aligned}
g & =\beta\left(\frac{\chi_{1}+\chi_{2}}{2}+(q+\delta) \frac{\chi_{1}-\chi_{2}}{2}\right) \\
& =\beta(1+q+\delta) \frac{\chi_{1}}{2}+\beta(1-q-\delta) \frac{\chi_{2}}{2}
\end{aligned}
$$


where $\delta= \pm \sqrt{1+q^{2}}$. A computation based on (2.3) and the linear independence of different characters shows that

$$
\begin{aligned}
\gamma(1-q+\delta) \mu\left(\chi_{1}\right) & =\gamma \beta(1-q+\delta)+\gamma \beta(1+q+\delta) \\
& =\gamma \beta(2+2 \delta)
\end{aligned}
$$

and

$$
\begin{aligned}
\gamma(1+q-\delta) \mu\left(\chi_{2}\right) & =\gamma \beta(1+q-\delta)+\gamma \beta(1-q-\delta) \\
& =\gamma \beta(2-2 \delta)
\end{aligned}
$$

Since $q \neq 0$, then $q-1 \neq \delta \neq q+1$ and so we find, as we have $\gamma \neq 0$, that

$$
\mu\left(\chi_{1}\right)=\beta \frac{2+2 \delta}{1-q+\delta} \text { and } \mu\left(\chi_{2}\right)=\beta \frac{2-2 \delta}{1+q-\delta} .
$$

So we are in the case (5) of our statement.

(iii) There exist a continuous character $\chi$ of $G$ and a non-zero continuous function $a \in \mathcal{A}(G)$ such that

$$
f=\gamma \chi \text { and } g=\beta \chi(1+a) \text { or } \quad f=\gamma(1+a) \chi \quad \text { and } \quad g=\beta \chi .
$$

By (2.3), the first case gives $a=0$ and $\beta=\frac{\mu(\chi)}{2}$. So we are in the point (2). For the case $f=\gamma(1+a) \chi$ and $g=\beta \chi$ we find, using (2.3), that $\mu(a \chi)=\mu(\chi)=\beta \neq 0$. So we are in the case (7) of our statement.

Conversely, some computations prove that the formulas above for $f$ and $g$ define solutions of $(2.2)$.

In the following corollary we solve the functional equation

$$
f\left(x y z_{0}\right)=f(x) g(y)+g(x) f(y), x, y \in G,
$$

for a fixed constant $z_{0} \in G$.

Corollary 2.4. Let $G$ be a topological group. Assume that the pair $f, g \in$ $C(G)$ is a solution of Eq. (2.5). Then we have the following possibilities:

1) $f=0$ and $g$ is arbitrary in $C(G)$. 
2) There exist a constant $\gamma \in \mathbf{C}^{\star}$ and a continuous character $\chi$ of $G$ such that

$$
f=\gamma \chi \text { and } g=\frac{\chi\left(z_{0}\right)}{2} \chi
$$

3) There exist a constant $\gamma \in \mathbf{C}^{\star}$ and two continuous characters $\chi_{1}$ and $\chi_{2}$ of $G$, with $\chi_{2}\left(z_{0}\right)=-\chi_{1}\left(z_{0}\right)$, such that

$$
f=\frac{\gamma}{2}\left(\chi_{1}+\chi_{2}\right) \text { and } g=\frac{\chi_{1}\left(z_{0}\right)}{2}\left(\chi_{1}-\chi_{2}\right) .
$$

4) There exist a constant $c \in \mathbf{C}^{\star}$ and two different continuous characters $\chi_{1}$ and $\chi_{2}$ of $G$, with $\chi_{2}\left(z_{0}\right)=\chi_{1}\left(z_{0}\right)$, such that

$$
f=c\left(\chi_{1}-\chi_{2}\right) \text { and } g=\frac{\chi_{1}\left(z_{0}\right)}{2}\left(\chi_{1}+\chi_{2}\right) .
$$

5) There exist constants $\gamma, \beta, q \in \mathbf{C}^{\star}$ and two continuous characters $\chi_{1}$ and $\chi_{2}$ of $G$, with $\chi_{1}\left(z_{0}\right)=\beta \frac{2+2 \delta}{1-q+\delta}$ and $\chi_{2}\left(z_{0}\right)=\beta \frac{2-2 \delta}{1+q-\delta}$, such that

$$
\begin{aligned}
f & =\gamma\left\{\frac{\chi_{1}+\chi_{2}}{2}+(-q+\delta) \frac{\chi_{1}-\chi_{2}}{2}\right\} \\
\text { and } g & =\beta\left\{\frac{\chi_{1}+\chi_{2}}{2}+(q+\delta) \frac{\chi_{1}-\chi_{2}}{2}\right\},
\end{aligned}
$$

where $\delta:= \pm \sqrt{1+q^{2}}$.

6) There exist a continuous function $a \in \mathcal{A}(G)$ and a continuous character $\chi$ of $G$, with $a\left(z_{0}\right)=0$, such that

$$
f=\chi a \text { and } g=\chi\left(z_{0}\right) \chi
$$

7) There exist a constant $\gamma \in \mathbf{C}^{\star}$, a continuous function $a \in \mathcal{A}(G)$ and a continuous character $\chi$ of $G$, with $a\left(z_{0}\right)=1$, such that

$$
f=\gamma(1+a) \chi \quad \text { and } g=\chi\left(z_{0}\right) \chi
$$

Conversely, the formulas above for $f$ and $g$ define solutions of (2.5). 
Proof. As the proof of Theorem 2.3 with $\mu=\delta_{z_{0}}$ and the fact that $\chi\left(z_{0}\right) \neq 0$.

Let $n \in \mathbf{N}, \alpha_{1}, \cdots, \alpha_{n} \in \mathbf{C}$, and $z_{1}, \cdots, z_{n} \in G$ be arbitrarily fixed elements. As another consequence of our result, in the following corollary, we obtain all continuous solutions of the following version of the sine addition formula

$$
\sum_{i=1}^{n} \alpha_{i} f\left(x y z_{i}\right)=f(x) g(y)+g(x) f(y), \quad x, y \in G .
$$

Corollary 2.5. Let $G$ be a topological group. Assume that the pair $f, g \in$ $C(G)$ is a solution of Eq. (2.6). Then we have the following possibilities:

1) $f=0$ and $g$ is arbitrary in $C(G)$ or $g=0$ and $f$ is any function in $C(G)$ such that $\sum_{i=1}^{n} \alpha_{i} f\left(x z_{i}\right)=0$ for all $x \in G$.

2) There exist a constant $\gamma \in \mathbf{C}^{\star}$ and a continuous character $\chi$ of $G$ with $\sum_{i=1}^{n} \alpha_{i} x\left(z_{i}\right) \neq 0$ such that

$$
f=\gamma \chi \text { and } g=\frac{1}{2} \frac{\sum_{i=1}^{n} \alpha_{i} \chi\left(z_{i}\right)}{2} \chi .
$$

3) There exist a constant $\gamma \in \mathbf{C}^{\star}$ and two different continuous characters $\chi_{1}$ and $\chi_{2}$ of $G$, with $\sum_{i=1}^{n} \alpha_{i} \chi_{2}\left(z_{i}\right)=-\sum_{i=1}^{n} \alpha_{i} \chi_{1}\left(z_{i}\right) \neq 0$, such that

$$
f=\frac{\gamma}{2}\left(\chi_{1}+\chi_{2}\right) \text { and } g=\frac{\sum_{i=1}^{n} \alpha_{i} \chi_{1}\left(z_{i}\right)}{2}\left(\chi_{1}-\chi_{2}\right) .
$$

4) There exist a constant $c \in \mathbf{C}^{\star}$ and two different continuous characters $\chi_{1}$ and $\chi_{2}$ of $G$, with $\sum_{i=1}^{n} \alpha_{i} \chi_{2}\left(z_{i}\right)=\sum_{i=1}^{n} \alpha_{i} \chi_{1}\left(z_{i}\right) \neq 0$, such that

$$
f=c\left(\chi_{1}-\chi_{2}\right) \text { and } g=\frac{\sum_{i=1}^{n} \alpha_{i} \chi_{1}\left(z_{i}\right)}{2}\left(\chi_{1}+\chi_{2}\right) \text {. }
$$

5) There exist constants $\gamma, \beta, q \in \mathbf{C}^{\star}$ and two continuous characters $\chi_{1}$ and $\chi_{2}$ of $G$, with $\sum_{i=1}^{n} \alpha_{i} \chi_{1}\left(z_{i}\right)=\beta \frac{2+2 \delta}{1-q+\delta}$ and $\sum_{i=1}^{n} \alpha_{i} \chi_{2}\left(z_{i}\right)=$ $\beta \frac{2-2 \delta}{1+q-\delta}$, such that

$$
f=\gamma\left\{\frac{\chi_{1}+\chi_{2}}{2}+(-q+\delta) \frac{\chi_{1}-\chi_{2}}{2}\right\} \text { and }
$$




$$
g=\beta\left\{\frac{\chi_{1}+\chi_{2}}{2}+(q+\delta) \frac{\chi_{1}-\chi_{2}}{2}\right\}
$$

where $\delta:= \pm \sqrt{1+q^{2}}$.

6) There exist a non-zero continuous function $a \in \mathcal{A}(G)$ and a continuous character $\chi$ of $G$, with $\sum_{i=1}^{n} \alpha_{i} a\left(z_{i}\right) \chi\left(z_{i}\right)=0$ and $\sum_{i=1}^{n} \alpha_{i} \chi_{1}\left(z_{i}\right) \neq 0$, such that

$$
f=\chi a \text { and } g=\sum_{i=1}^{n} \alpha_{i} \chi\left(z_{i}\right) \chi
$$

7) There exist a constant $\gamma \in \mathbf{C}^{\star}$, a continuous function $a \in \mathcal{A}(G)$ and a continuous character $\chi$ of $G$, with $\sum_{i=1}^{n} \alpha_{i} \chi\left(z_{i}\right)=\sum_{i=1}^{n} \alpha_{i} a\left(z_{i}\right) \chi\left(z_{i}\right) \neq$ 0 , such that

$$
f=\gamma(1+a) \chi \quad \text { and } g=\sum_{i=1}^{n} \alpha_{i} \chi\left(z_{i}\right) \chi
$$

Conversely, the formulas above for $f$ and $g$ define solutions of (2.6).

Proof. As the proof of Theorem 2.3 with $\mu=\sum_{i=1}^{n} \alpha_{i} \delta_{z_{i}}$.

In the following proposition we show that the integral functional equation for the sine subtraction law

$$
\int_{G} f(x y t) d \mu(t)=f(x) g(y)-g(x) f(y), x, y \in G,
$$

has only "trivial" solutions.

Proposition 2.6. Let $G$ be a locally compact Hausdorff group. The pair $f, g \in C(G)$ is a solution of Eq. (2.7) if and only if it has one of the following forms:

(i) $f=0$ and $g$ is arbitrary in $C(G)$.

(ii) There exists a constant $\alpha \in \mathbf{C}$ such that $g=\alpha f$ and $f$ is any function in $C(G)$ such that $\int_{G} f(\cdot t) d \mu(t)=0$. 
Proof. Let the pair $f, g$ be a solution of the equation (2.7). It is easy to see that

$$
\int_{G} f(y x t) d \mu(t)=-\int_{G} f(x y t) d \mu(t) \text { for all } x, y \in G .
$$

Letting $y=e$ in (2.8) we get that $\int_{G} f(x t) d \mu(t)=0$ for all $x \in G$. Thus we get that

$$
f(x) g(y)=g(x) f(y), \text { for all } x, y \in G .
$$

If $f=0$ then we are in (i) and if $f \neq 0$ then $g=\alpha f$ where $\alpha=\frac{g(a)}{f(a)}$ for some $a \in G$ such that $f(a) \neq 0$ then we are in (ii). Conversely, the formulas above for $f$ and $g$ define solutions of (2.7).

\section{An example}

Example 3.1. Let $G=(\mathbf{R},+), z_{0} \in \mathbf{R}^{\star}$ be a fixed element, and let $\mu=$ $\delta_{z_{0}}$.

We indicate here the corresponding continuous solutions of Eq. (2.2) (i.e. (2.5)) by the help of Corollary 2.4.

The continuous characters on $\mathbf{R}$ are known to be $\chi(x)=e^{\lambda x}, x \in \mathbf{R}$, where $\lambda$ ranges over $\mathbf{C}$ (see for instance [8, Example 3.7(a)]).

The continuous additive functions on $\mathbf{R}$ are the functions of the form $a(x)=\alpha x, x \in \mathbf{R}$, where the constant $\alpha$ ranges over $\mathbf{C}$ (see for instance $[8$, Corollary 2.4]). In the point (6) of Corollary 2.4 we have $a\left(z_{0}\right)=0$ which reduces to $\alpha=0$ i.e. $a=0$. So this case does not occur here. In the point (4) of Corollary 2.4 we have $\chi_{2}\left(z_{0}\right)=\chi_{1}\left(z_{0}\right)$ which reduces to $e^{\lambda_{1} z_{0}}=e^{\lambda_{2} z_{0}}$ for some $\lambda_{1}, \lambda_{2} \in \mathbf{R}$ then $\lambda_{1}=\lambda_{2}$ i.e. $\chi_{1}=\chi_{2}$. So this case does not occur here either.

In conclusion, by help of Corollary 2.4 we find that the continuous solutions $f, g: \mathbf{R} \rightarrow \mathbf{C}$ of the functional equation (2.2), which is here

$$
f\left(x+y+z_{0}\right)=f(x) g(x)+f(y) g(x), x, y \in \mathbf{R},
$$

are

1. $f=0$ and $g$ is any continuous function.

2 .

$$
f(x)=\gamma e^{\lambda x} \text { and } g(x)=\frac{1}{2} e^{\lambda\left(x+z_{0}\right)}, x \in \mathbf{R},
$$

for some constants $\gamma \in \mathbf{C}^{\star}, \lambda \in \mathbf{C}$. 
3.

$$
f(x)=\frac{\gamma}{2}\left(e^{\lambda_{1} x}+e^{\lambda_{2} x}\right) \text { and } g(x)=\frac{1}{2}\left(e^{\lambda_{1}\left(x+z_{0}\right)}+e^{\lambda_{2}\left(x+z_{0}\right)}\right), x \in \mathbf{R},
$$

for some constants $\gamma \in \mathbf{C}^{\star}$ and $\lambda_{1}, \lambda_{2} \in \mathbf{C}$ such that $e^{\left(\lambda_{2}-\lambda_{1}\right) z_{0}}=-1$.

4 .

$$
\begin{gathered}
f(x)=\gamma\left(\frac{e^{\lambda_{1} x}+e^{\lambda_{2} x}}{2}+(\delta-q) \frac{e^{\lambda_{1} x}-e^{\lambda_{2} x}}{2}\right) \text { and } \\
g(x)=\beta\left(\frac{e^{\lambda_{1} x}+e^{\lambda_{2} x}}{2}+(\delta+q) \frac{e^{\lambda_{1} x}-e^{\lambda_{2} x}}{2}\right),
\end{gathered}
$$

for some constants $\gamma, \beta, q \in \mathbf{C}^{\star}, \lambda_{1}, \lambda_{2} \in \mathbf{C}$ and $\delta:= \pm \sqrt{1+q^{2}}$ such that $e^{\lambda_{1} z_{0}}=\beta \frac{2+2 \delta}{1-q-\delta}$ and $e^{\lambda_{2} z_{0}}=\beta \frac{2-2 \delta}{1+q-\delta}$.

5.

$$
f(x)=\gamma\left(1+\frac{x}{z_{0}}\right) e^{\lambda x} \text { and } g(x)=e^{\lambda\left(x+z_{0}\right)}, x \in \mathbf{R},
$$

for some constants $\gamma \in \mathbf{C}^{\star}, \lambda \in \mathbf{C}$.

Acknowledgement. The authors would like to express their most sincere gratitude to the referee for a number of constructive comments which have led to essential improvement of the paper.

\section{References}

[1] J. Aczél, Lectures on Functional Equations and Their Applications. Mathematics in Science and Engineering, vol. 19. Academic Press, New York, $\mathrm{xx}+510,(1966)$.

[2] J. K. Chung, Pl. Kannappan, C. T. Ng, A generalization of the cosinesine functional equation on groups. Linear Algebra Appl. 66, pp. 259$277,(1985)$.

[3] B. R. Ebanks, H. Stetkær, d'Alembert's other functional equation on monoids with an involution. Aequationes Math. 89 (1), pp. 187-206, (2015). 
[4] B. Fadli, D. Zeglami, S. Kabbaj, The generalized Van Vleck's equation on locally compact groups. Proyecciones J. of Math., 36 (4), pp. 545$566,(2017)$.

[5] B. Fadli, D. Zeglami, S. Kabbaj, An integral functional equation on groups under two measures. Proyecciones J. of Math., 37 (3), pp. 565$581,(2018)$.

[6] Pl. Kannappan, Functional Equations and Inequalities with Applications. Springer, New York, 39-02 (39Bxx), (2009).

[7] Th. A. Poulsen, H. Stetkær, On the trigonometric subtraction and addition formulas. Aequationes Math. 59 (1), pp. 84-92 (2000).

[8] H. Stetkær, "Functional equations on groups". World Scientific Publishing Company, Singapore, (2013).

[9] H. Stetkær, Van Vleck's functional equation for the sine. Aequationes Math. 90 (1), pp. 25-34, (2016).

[10] H. Stetkær, The cosine addition law with an additional term, Aequationes Math. 90 (6), pp. 1147-1168, (2016).

[11] L. Székelyhidi, Convolution Type Functional Equations on Topological Abelian Groups, World Scientific Publishing Company, Singapore-New Jersey-London-Hong Kong, (1991).

[12] E. B. Van Vleck, A functional equation for the sine. Ann. of Math., Second Series, 11 (4), pp. 161-165, (1910).

[13] D. Zeglami, B. Fadli, S. Kabbaj, Harmonic analysis and generalized functional equations for the cosine, Adv. Pure Appl. Math. 7 (1), pp. 41-49, (2016).

[14] D. Zeglami, B. Fadli, Integral functional equations on locally compact groups with involution, Aequationes Math. 90 (5), pp. 967-982, (2016).

[15] D. Zeglami, Some functional equations related to number theory, Acta Math. Hungar. 149, no. 2, pp. 490-508, (2016).

[16] D. Zeglami, M. Tial, S. Kabbaj, The integral cosine addition and sine subtraction laws, Results Math. 73, no.3, 97, (2018). 
D. Zeglami,

Department of Mathematics, E. N. S. A. M., Moulay Ismail University ,

B. P. : 15290 Al Mansour, Meknes, Morocco

e-mail : zeglamidriss@yahoo.fr

\section{Tial,}

Department of Mathematics,

Faculty of Sciences,

Ibn Tofail University,

BP: 14000 .

Kenitra,

Morocco

e-mail : tialmohamed@gmail.com

and

\section{S. Kabbaj}

Department of Mathematics,

Faculty of Sciences,

Ibn Tofail University,

BP: 14000 .

Kenitra,

Morocco

e-mail : samkabbaj@yahoo.fr 